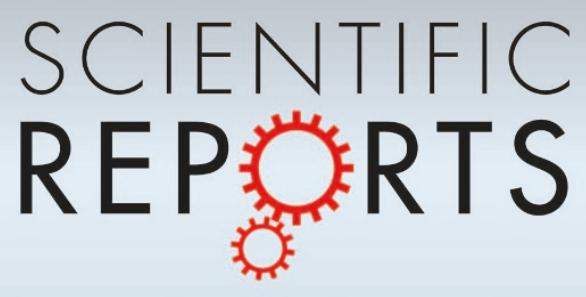

OPEN

SUBJECT AREAS:

GASTROINTESTINAL

CANCER

RISK FACTORS

PROGNOSTIC MARKERS

GASTROINTESTINAL DISEASES

Received

25 June 2013

Accepted

16 September 2013

Published

3 October 2013

Correspondence and requests for materials should be addressed to

Z.J. (zhejin1995@ yahoo.com) or S.J.M. (smeltzer@jhmi.edu)

* These authors contributed equally to this work.

\section{MAL hypermethylation is a tissue-specific event that correlates with MAL mRNA expression in esophageal carcinoma}

Zhe Jin 1,3,4*, Liang Wang ${ }^{1 *}$, Yuan Zhang ${ }^{1 *}$, Yulan Cheng ${ }^{2}$, Yan Gao ${ }^{5}$, Xianling Feng ${ }^{1}$, Ming Dong ${ }^{1}$, Ziyi Cao', Si Chen', Huimin Yu' ', Zhenfu Zhao', Xiaojing Zhang' ', Jie Liu', Yuriko Mori' ', Xinmin Fan' \& Stephen J. Meltzer²

\footnotetext{
'Department of Pathology, The Shenzhen University School of Medicine, Shenzhen, Guangdong, People's Republic of China, ${ }^{2}$ Division of Gastroenterology, Department of Medicine, The Johns Hopkins University School of Medicine and Sidney Kimmel Comprehensive Cancer Center, Baltimore, Maryland, USA, ${ }^{3}$ Shenzhen Key Laboratory of Micromolecule Innovatal Drugs, Shenzhen, Guangdong, People's Republic of China, ${ }^{4}$ Laboratory of Chemical Genomics, School of Chemical Biology and Biotechnology, Peking University Shenzhen Graduate School, Shenzhen, Guangdong, People's Republic of China, ${ }^{5}$ Nanshan Hospital, Guangdong Medical College, Shenzhen.
}

MAL promoter hypermethylation was examined in 260 human esophageal specimens using real-time quantitative methylation-specific PCR (qMSP). MAL hypermethylation showed highly discriminative ROC curve profiles which clearly distinguished esophageal adenocarcinomas (EAC) from both esophageal squamous cell carcinomas (ESCC) and normal esophagus (NE). Both MAL methylation frequency and normalized methylation value (NMV) were significantly higher in Barrett's esophagus (BE), dysplastic BE, and EAC than in ESCC or in NE. Among matched NE and EAC samples, MAL NMVs in EAC were significantly higher than in corresponding NE. There was a significant correlation between MAL hypermethylation and BE segment length. Treatment with 5-aza-2' -deoxycytidine reversed $M A L$ methylation and reactivated $M A L$ mRNA expression in OE33 EAC cells. MAL mRNA levels in EACs with unmethylated MAL were significantly higher than in EACs with methylated MAL. MAL hypermethylation is a common, tissue-specific event in human EAC and correlates with clinical neoplastic progression risk factors.

\footnotetext{
E sophageal cancer ranks as the $8^{\text {th }}$ most-frequent and $6^{\text {th }}$ most-fatal cancer type worldwide, with an estimated 480,000 new cases diagnosed and 400,000 deaths globally in $2008^{1}$. This cancer consist of two major histologic subtypes: esophageal adenocarcinoma (EAC), which is more prevalent in Western countries, with a rapidly increasing incidence; and esophageal squamous cell carcinoma (ESCC), which is frequent in developing countries, especially in Asia, and including China ${ }^{2}$. Since both types of esophageal cancer exhibit highly aggressive behavior, with rapid progression to death ${ }^{3}$, a better understanding of the molecular events underlying their pathogenesis is essential to achieving improved survival. Therefore, we sought to discover molecular events with potential asearly detection biomarkers ortargets of chemoprevention and therapy.

Myelin and Lymphocyte protein (MAL, also known as mal or T-cell differentiation protein), a $17 \mathrm{kDa}$ hydrophobic membrane protein, is widely expressed in a variety of cell types, including T-lymphocytes ${ }^{4}$, myelin-forming cells $s^{5,6}$, and epithelial cells of the kidney, stomach, and large intestine ${ }^{7,8}$. It has recently been clarified that MAL constitutes an essential component of glycolipid-enriched membrane micro-domains or lipid rafts involved in the apical transport of membrane and secretory proteins in polarized epithelial cells ${ }^{9,10}$. Apical transport is essential for the proper functioning of epithelial cells, and the neoplastic transformation process is frequently associated with loss of this polarized phenotype ${ }^{11}$. Several investigations have indicated that downregulation of MAL may constitute as a common molecular event contributing to the initiation and/or progression of several cancers, including those arising in the digestive tract. For example, using tissue microarrays, Guro et al. found that epithelial cells of colorectal carcinomas were MAL-negative, whereas in normal colonic tissues cytoplasmic expression of MAL occurred in both epithelial and connective tissues ${ }^{12}$. Koshi et al. showed that MAL expression was reduced or absent in esophageal cancers $v s$. normal cells, using differential display experiments $^{13}$. Dysregulation of MAL has also been implicated in several other malignancies, including breast ${ }^{14}$, cervical $^{15}$ and HNSCC cancers $^{16}$. These results suggest that MAL possesses tumour-suppressive capabilities.
} 

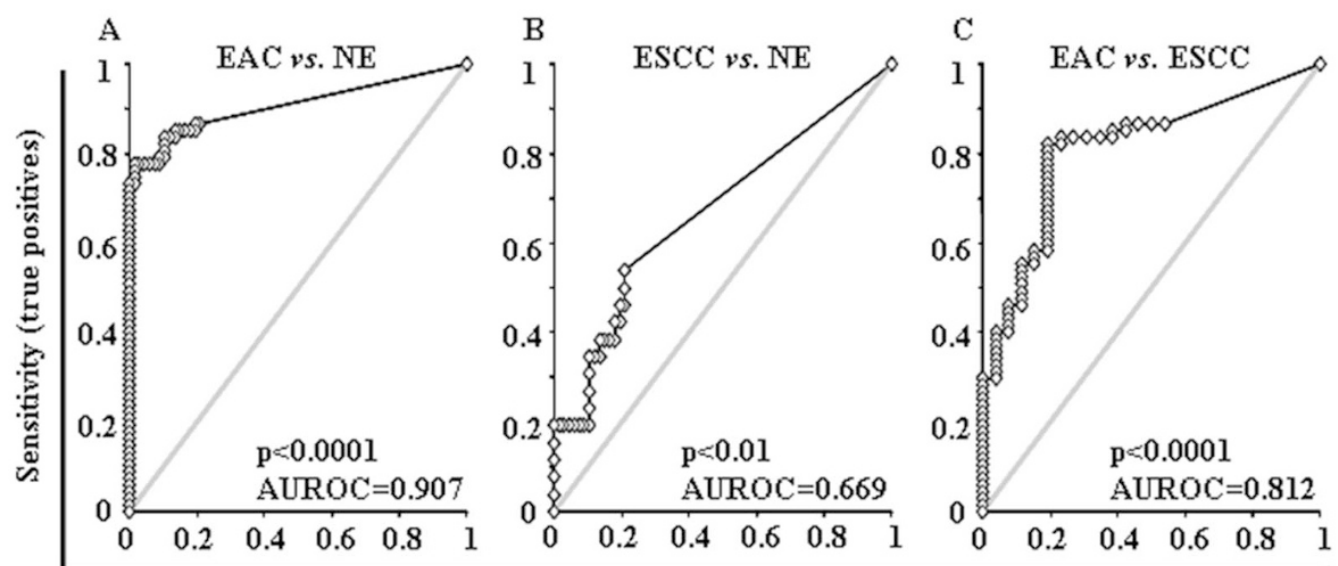

1-Specificity (false positives)

$$
\text { No discrimination } \prec \text { MAL }
$$

Figure 1 Receiver-operator characteristic (ROC) curve analysis of normalized methylation value (NMV). ROC curve analysis of MAL NMVs of normal esophagus (NE) vs. esophageal adenocarcinoma (EAC) (A), NE vs. esophageal squamous cell carcinoma (ESCC) (B), and EAC vs. ESCC (C). The area under the ROC curve (AUROC) conveys this biomarker's accuracy in distinguishing EAC from NE and from ESCC in terms of its sensitivity and specificity.

Aberrant methylation of promoter $\mathrm{CpG}$ islands upstream of tumor suppressor genes is now well-established as a major mechanism of gene inactivation in human tumorigenesis ${ }^{17}$, including ESCC and $\mathrm{EAC}^{18}$, where it plays an important role in pathogenesis ${ }^{19-22}$. Some of these methylation events appear to represent useful prognostic markers, as they precede and thus predict the progression of Barrett's esophagus (BE) to $\mathrm{EAC}^{23}$. Aberrant promoter methylation of $M A L$ is indeed associated with inactivation of its expression in breast and colorectal cancers ${ }^{12,14}$. Therefore, we hypothesized that $M A L$ might be inactivated via promoter hypermethylation in human esophageal cancers, and that hypermethylation of $M A L$ could constitute an early event in the genesis of EAC.

\section{Results}

MAL promoter hypermethylation in different esophageal tissues. Promoter hypermethylation of the $M A L$ gene was analyzed in 67 normal esophagus (NE), $60 \mathrm{BE}, 40$ dysplasias occurring in $\mathrm{BE}[\mathrm{D}$, 19 low-grade (LGD) and 21 high-grade (HGD)], 67 EAC and 26 ESCC samples. MAL promoter hypermethylation showed highly discriminative receiver-operator characteristic (ROC) curve profiles, which clearly distinguished EAC from both NE and ESCC. ROC curves with corresponding the area under the ROC curve
(AUROC) for MAL of EAC vs. NE, ESCC vs. NE and EAC vs. ESCC are shown in Figure 1.

The cutoff normalized methylation value (NMV) for MAL (0.02) was chosen from ROC curves to maximize sensitivity and specificity. Mean NMV and frequency of MAL hypermethylation for each tissue type are shown in Table 1 . The mean NMV of $M A L$ was significantly higher in BE (0.0681, p = 0000001), LGD (0.0945, p = 000004), HGD (0.0549, $\mathrm{p}=0000001), \mathrm{D}$ (0.0737, $\mathrm{p}=00000001)$, EAC (0.0459, $\mathrm{p}=0.000002)$, and ESCC $(0.0042, \mathrm{p}=0.009)$ than in NE (0.0001; Student's t-test). The frequency of $M A L$ hypermethylation was increased in BE (53.3\%), LGD (63.2\%), HGD (57.1\%), D (60\%), and EAC (40.3\%) vs. NE (0.0\%; all p < 0.01; Fisher's exact probability test). Among 28 cases with matched NE and EAC, the MAL NMVs in EAC $($ mean $=0.0610)$ were significantly higher than in corresponding NE (mean $=0.0001 ; \mathrm{p}=0.0015$, Student's paired t-test; Figure 2A). However, Among 13 cases with matched $\mathrm{NE}$ and ESCC, the difference of MAL NMVs in ESCC (mean $=0.0023$ ) and in corresponding NE (mean $=0.0003$ ) were not significant ( $\mathrm{p}$ $=0.29$, Student's paired t-test; Figure 2B). Finally, both NMVs and hypermethylation frequency of $M A L$ were significantly higher in $\mathrm{BE}$, $\mathrm{D}$ and EAC than in ESCC (Table 1).

According to generally accepted criteria ${ }^{24}, \mathrm{BE}$ was defined as longsegment (LSBE) if it was equal to or greater than $3 \mathrm{~cm}$ in length, or

\begin{tabular}{|c|c|c|c|c|c|c|}
\hline Histological type & samples & mean & $M / F$ & W/B/A/unknown & mean $\pm S D *$ & cut off $0.02 * *$ \\
\hline $\begin{array}{l}\text { Normal esophagus } \\
\text { Barrett's metaplasia } \\
\text { Dysplasia in Barrett's esophagus } \\
\text { Low-grade dysplasia } \\
\text { High-grade dysplasia } \\
\text { Esophageal adenocarcinoma } \\
\text { Esophageal squamous cell } \\
\text { carcinoma }\end{array}$ & $\begin{array}{l}67 \\
60 \\
40 \\
19 \\
21 \\
67 \\
26\end{array}$ & $\begin{array}{l}64.4 \\
63.7 \\
65.3 \\
65.3 \\
65.2 \\
65.1 \\
62.5\end{array}$ & $\begin{array}{l}60 / 7 \\
56 / 4 \\
35 / 5 \\
17 / 2 \\
18 / 3 \\
58 / 9 \\
19 / 7\end{array}$ & $\begin{array}{l}40 / 11 / 4 / 12 \\
43 / 3 / 1 / 13 \\
29 / 3 / 2 / 6 \\
13 / 2 / 1 / 3 \\
16 / 1 / 1 / 3 \\
57 / 1 / 2 / 7 \\
8 / 13 / 2 / 3\end{array}$ & $\begin{array}{l}0.00014 \pm 0.00039 \\
0.0681 \pm 0.09951 \# / \$ \\
0.0737 \pm 0.11781^{\# / \$} \\
0.0945 \pm 0.15812^{\# / \$} \\
0.0549 \pm 0.06149^{\# / \$} \\
0.0459 \pm 0.07453^{\# / \$} \\
0.0042 \pm 0.01248^{\#}\end{array}$ & $\begin{array}{c}0 \\
53.3 \%(32 / 60)^{\# / \$} \\
60 \%(24 / 40)^{\# / \$} \\
63.2 \%(12 / 19)^{\# / \$} \\
57.1 \%(12 / 21)^{\# / \$} \\
40.3 \%(27 / 67)^{\# / \$} \\
7.7 \%(2 / 26)\end{array}$ \\
\hline
\end{tabular}




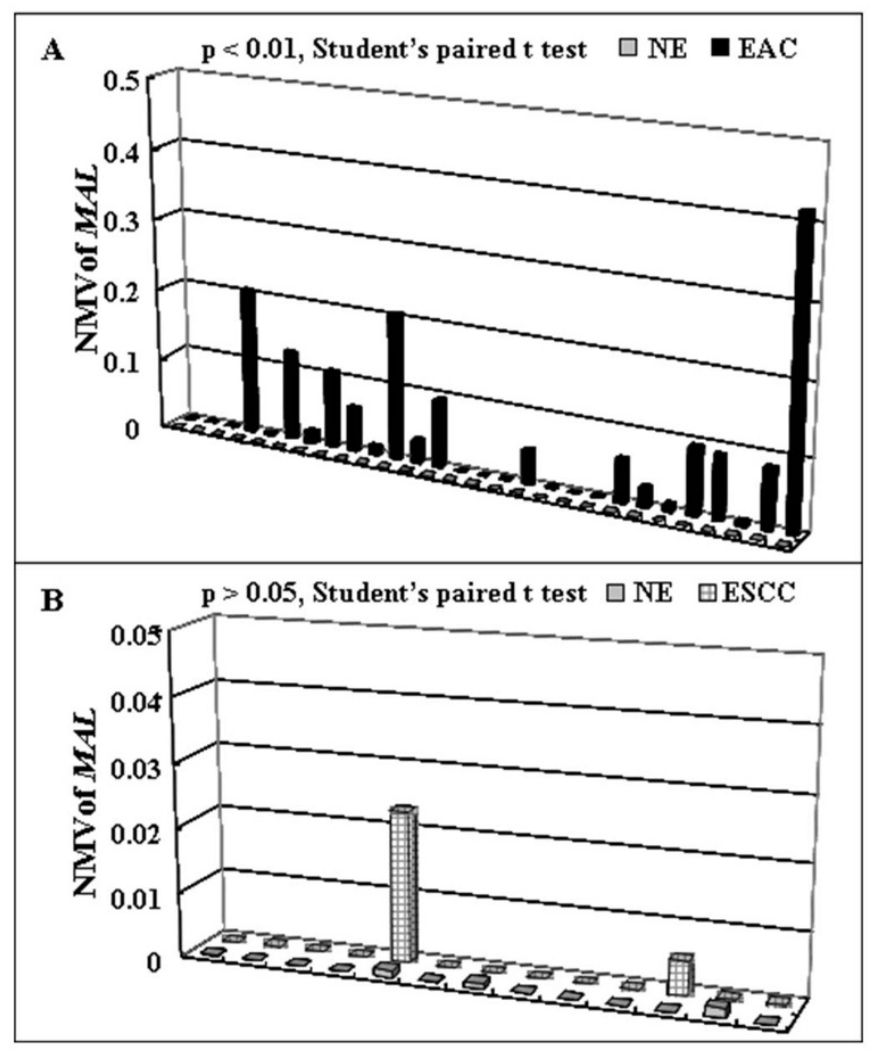

Figure $2 \mid$ Methylation status of $M A L$ in corresponding esophageal samples. (A) In 28 patients with corresponding NE and EAC. MAL normalized methylation values for EAC $($ mean $=0.0610)$ were significantly higher than those for matching NE ( mean $=0.0001 ; \mathrm{p}<0.01$, Student $t$ test for paired data). (B) In 13 patients with corresponding NE and ESCC, the differences between MAL NMVs for ESCC ( mean $=0.0023)$ and those for matching NE ( mean $=0.0003)$ were not significantly $(\mathrm{p}>$ 0.05 , Student $t$ test for paired data).

short-segment (SSBE) if less than $3 \mathrm{~cm}$. The mean NMV of $M A L$ was significantly higher in LSBE (mean $=0.1235)$ than in SSBE $($ mean $=$ 0.0269; $\mathrm{p}=0.03$, Student's t-test, Figure 3A). Similarly, segment lengths of BEs with hypermethylated $M A L$ promoters (mean =

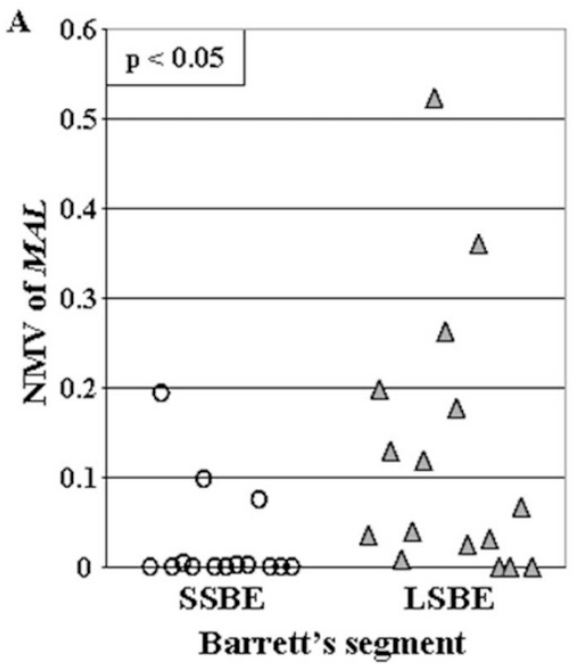

$5.87 \mathrm{~cm}$ ) were significantly greater than segment lengths of BEs with unmethylated $M A L$ promoters (mean $=2.60 \mathrm{~cm} ; \mathrm{p}=0.005$, Student's t-test; Figure 3B).

No significant associations were observed between $M A L$ promoter hypermethylation and patient age, survival, smoking or alcohol consumption status, tumor stage or lymph node metastasis, histologic tumor differentiation, or histologic type of esophageal carcinoma (data not shown).

MAL methylation and mRNA levels in OE33 cells after 5-Aza-dC treatment. OE33 cells were subjected to demethylation by 5 -Aza$\mathrm{dC}$ treatment. After 5-Aza-dC treatment, the NMV of MAL was diminished $(0.4264,0.1647$ and 0.1414 on day 0,2 and 4 , respectively), while the mRNA level of $M A L(0.0184$ on day 0$)$ was increased on day $2(0.0643)$ and further increased on day $4(0.0773$, Figure 4).

Inverse correlation between hypermethylation and mRNA expression of MAL in human esophageal tissues. To further elucidate the relationship between DNA hypermethylation and mRNA expression of $M A L$, we determined the normalized mRNA value (NRV) of $M A L$ in $10 \mathrm{NE}$ and 28 EAC samples using qRT-PCR. The mean NRV of $M A L$ in EAC (mean $=0.0819)$ was significantly lower than in NE $($ mean $=0.4128 ; \mathrm{p}=0.00015$, Mann-Whitney U test; Figure 5A). The mean NRV of MAL in specimens with unmethylated $M A L$ (mean $=0.2418, \mathrm{n}=24$ ) was significantly higher than in specimens with hypermethylated $M A L$ (mean $=0.0441, \mathrm{n}=14 ; \mathrm{p}$ $=0.0084$, Mann-Whitney U test; Figure 5B). A significant negative correlation (Pearson correlation coefficient $\mathrm{r}=-0.363, \mathrm{p}=0.025$ ) between NMVs and NRVs of MAL was also found among these samples (Figure 5C).

\section{Discussion}

$M A L$ down-regulation has been reported in many types of cancer, including breast, cervical, colorectal and esophagus ${ }^{14,15,25,26}$. These results suggest that low expression of $M A L$ may represent a general characteristic or even a requirement of transformed cells in many kinds of carcinogenesis. Potential mechanisms underlying this suppression of expression include posttranscriptional and epigenetic changes, such as aberrant DNA methylation ${ }^{20,27}$. Koshi et al. reported that the expression of MAL was extinguished in ESCC, and speculated that DNA methylation and histone deacetylation in the $M A L$

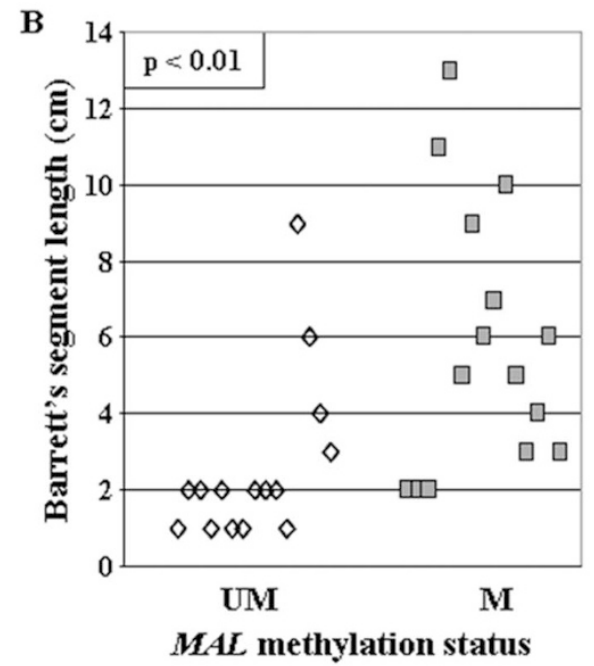

Figure 3 Correlation between Barrett's segment length and MAL hypermethylation. (A) Mean NMV of MAL was significantly higher in LSBE (mean $=0.1235$, black triangle) than in SSBE (mean $=0.0269$, white cricle; $\mathrm{p}<0.05$, Student's t test). (B) segment lengths of BEs with hypermethylated $M A L$ promoters $($ mean $=5.87 \mathrm{~cm}$, black square) were significantly greater than segment lengths of BEs with unmethylated $M A L$ promoters $($ mean $=$ $2.60 \mathrm{~cm}$, white rhombus; $\mathrm{p}<0.01$, Student's t-test). 


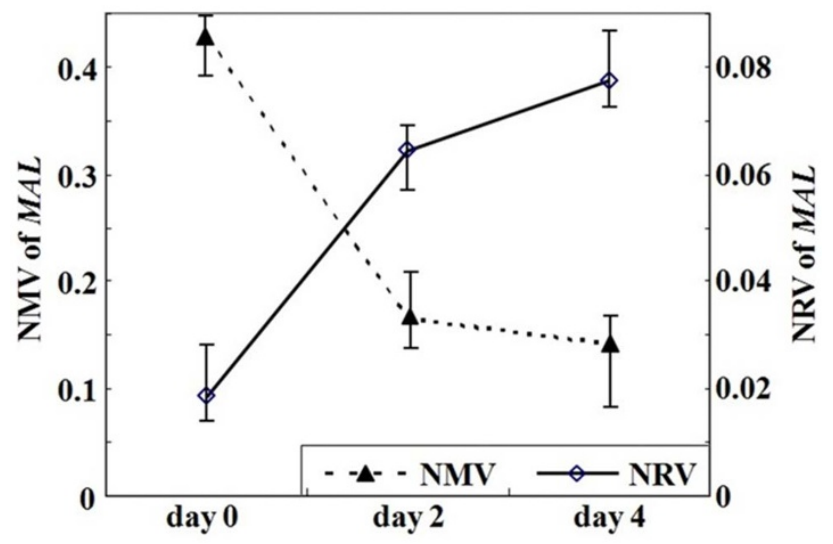

Figure $4 \mid M A L$ methylation level and mRNA expression in esophageal cancer cell line (OE33) after treatment with the demethylating agent 5-aza-20-deoxycytidine (5-Aza-dC). After 5- Aza-dC treatment, the NMV of MAL was diminished, while the NRV of MAL was increased.

promoter may act as synergistic controls to silence the MAL gene in esophageal cancer ${ }^{13}$. However, evidence for this conclusion was based on 5-aza-20-deoxycytidine and trichostatin A-treated esophageal carcinoma cell lines. Whether hypermethylation of the $M A L$ gene was a common event in esophageal carcinoma was thus still unknown. Further evidence based on sufficient clinical samples was therefore needed.

In the current study, by studying 260 primary human tissue specimens, we systematically investigated hypermethylation of the $M A L$ gene promoter in primary human esophageal lesions of differing histological types and grades using real-time quantitative methylation-specific PCR (qMSP). DNA methylation levels were not validated by other methods, such as pyrosequencing. Our results demonstrate that $M A L$ promoter hypermethylation occurs frequently in human EAC, but not in ESCC (Table 1). MAL NMVs in EAC were significantly higher than in corresponding NE in 28 cases with matched NE and EAC (Figure 2A). However, no significant difference of MAL NMVs was found between NE and ESCC in 13 cases with matched NE and ESCC (Figure 2B). In addition, both NMV and hypermethylation frequency of $M A L$ were significantly higher in BE, D and EAC than in ESCC (Table 1). These results suggest that hypermethylation of $M A L$ may represent an early epigenetic event in these subjects, and that this event is highly prevalent in human esophageal adenocarcinomas.

$\mathrm{BE}$ is the major risk factor for esophageal adenocarcinoma ${ }^{28}$. Although it is somewhat arbitrary to separate SSBE from LSBE, this distinction persists in clinical practice ${ }^{29,30}$. To date, length of BE as a predictive factor for $\mathrm{BE}$ progression remains controversial. While

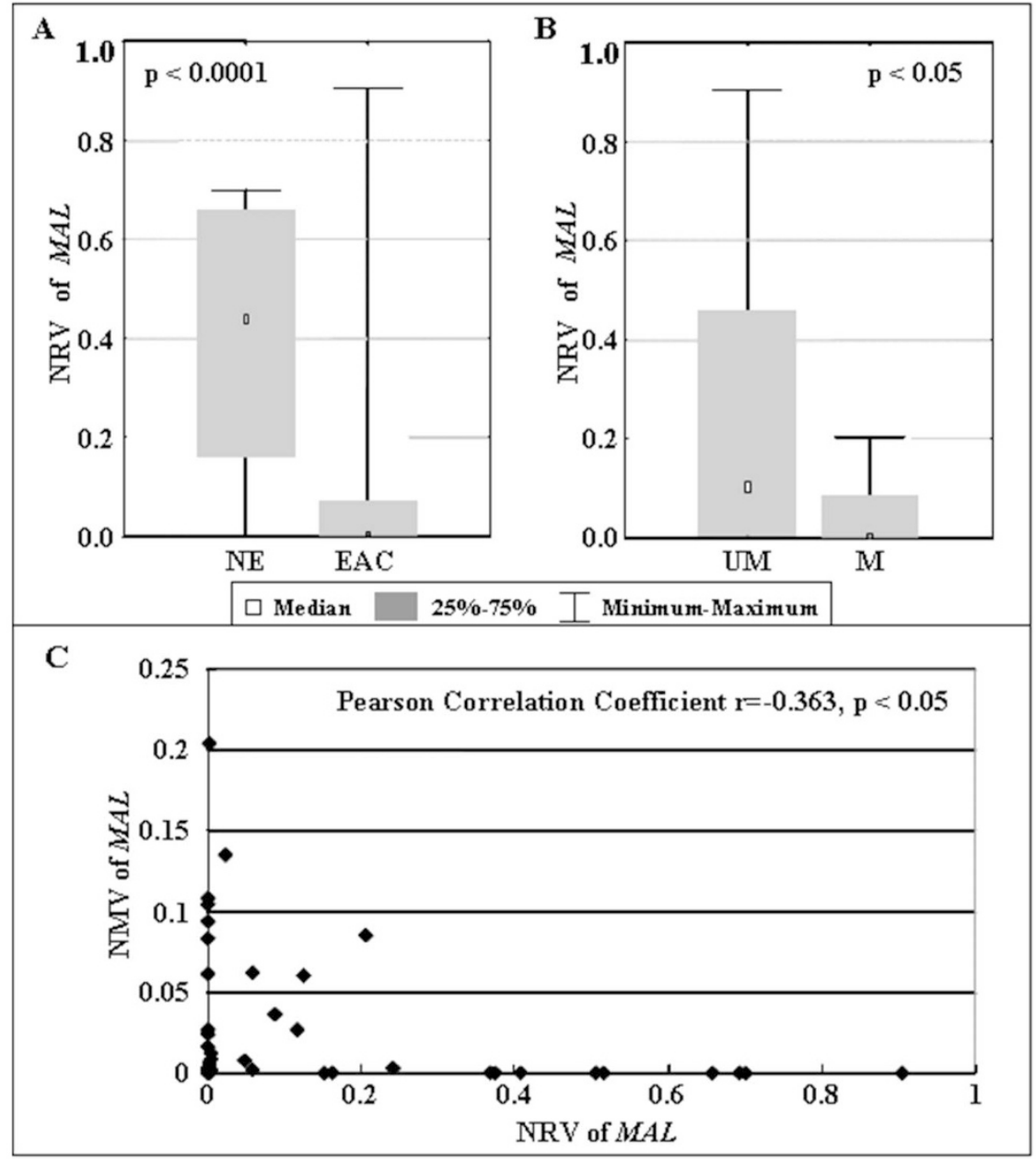

Figure $5 \mid$ Correlation between MAL methylation status and mRNA expression levels in human esophageal tissues. (A) The mean NRV of MAL in EAC $($ mean $=0.0819, \mathrm{n}=28$ ) was significant lower than in NE (mean $=0.4128, \mathrm{n}=10 ; \mathrm{p}<0.0001$, Mann-Whitney U test). (B) The mean NRV of MAL in specimens with unmethylated MAL (mean $=0.2418, \mathrm{n}=24)$ was significantly higher than in specimens with hypermethylated MAL $($ mean $=$ $0.0441, \mathrm{n}=14 ; \mathrm{p}<0.05$, Mann-Whitney U test). (C) Significant negative correlation (Pearson correlation coefficient $\mathrm{r}=-0.363, \mathrm{p}<0.05$ ) between NMV and NRV of MAL in human esophageal tissues. 
several previous studies found that patients with SSBE can develop dysplasia and cancer $^{31,32}$, the statement that an increased risk of EAC development exists only in patients with LSBE $^{33,34}$ is not accepted universally. Giovanni et al. reported that complete-type intestinal metaplasia (IM), which is considered the least prone to cancerization, was significantly prevalent in SSBE patients at baseline, whereas the incomplete type of IM, which is more closely associated with cancer progression, significantly prevailed in $\mathrm{LSBE}^{35}$. In a prospective cohort study of 309 Barrett's patients followed in the Seattle Barrett's Esophagus Project, a strong trend was observed, with a $5 \mathrm{~cm}$ difference in length associated with a 1.7 -fold increase in cancer risk (95\% CI, 0.8-3.8-fold), when patients with HGD at entrance were excluded $^{36}$. Several other researchers also demonstrated significant differences between SSBE and LSBE in the frequency of both dysplasia and $\mathrm{EAC}^{33,34}$. Thus, it is likely that length of Barrett's epithelium is an important risk factor for both the prevalence of concurrent dysplasia or cancer and the incidence of future malignant progression. Notably, MAL hypermethylation exhibited a strong correlation with $\mathrm{BE}$ segment length in the current study (Figure 3). Therefore, $M A L$ methylation may constitute both a molecular correlate of BE segment length, and a potential biomarker for the prediction of future $\mathrm{BE}$ progression.

We further analyzed the methylation status and mRNA expression level of MAL, both in 5-Aza-dC treated EAC cells and in primary human EAC specimens. These experiments revealed that reversal of methylation and restoration of $M A L$ expression were induced in OE33 cells by 5 -Aza-dC treatment (Figure 4). Restoration of $M A L$ mRNA expression due to 5-Aza-dC treatment is consistent with the interpretation that DNA promoter hypermethylation silences the $M A L$ gene. This experiment was performed in only one EAC cell line, and not in any BE or ESCC cell lines. Moreover, in 38 esophageal specimens, the mean NRV of $M A L$ was significantly higher in specimens with unmethylated $M A L$ promoters than in specimens with hypermethylated $M A L$ (Figure 5B), and a significant negative correlation between NMV and NRV of MAL was also observed (Figure 5C). It is worthwhile to note that many previous studies have proposed epigenetic regulation of $M A L$ expression ${ }^{12,37,38}$, These previous observations, together with our own new data, suggest that epigenetic regulation (such as promoter hypermethylation) is essential in silencing of $M A L$ expression in human esophageal adenocarcinoma, and that the $M A L$ gene is a candidate tumor suppressor gene in this disease.

In conclusion, the current study shows that hypermethylation of the $M A L$ gene promoter, leading to gene silencing, is a common event in human Barrett's-associated EAC and is associated with clinical risk factors of neoplastic progression. In addition, $M A L$ hypermethylation is uncommon in human ESCC, thus making this event a potential cell type-specific biomarker for EAC.

\section{Methods}

Tissue samples. In the current study, $67 \mathrm{NE}, 60 \mathrm{BE}$ samples without dysplasia, 19 lowgrade and 21 high-grade dysplasias occurring in BE (D), 67 EACs, and 26 ESCCs were examined. All patients provided written informed consent under a protocol approved by the Institutional Review Boards at the University of Maryland and Baltimore Veterans Affairs Medical Centers, where all esophagogastroduodenoscopies were performed. Biopsies were taken using a standardized biopsy protocol, as previously described ${ }^{19}$. Research tissues were obtained from grossly apparent Barrett's epithelium or from mass lesions in patients manifesting these changes at endoscopic examination, and histology was confirmed using parallel aliquots obtained at endoscopy. All biopsy specimens were stored in liquid nitrogen before DNA/RNA extraction. Clinicopathologic characteristics are summarized in Table 1.

Cell lines. The EAC (OE33) cell line was obtained from the ATCC (Manassas, VA). These cells were cultured in 47.5\% RPMI 1640, 47.5\% F-12 supplemented with 5\% fetal bovine serum.

DNA and RNA extraction. Genomic DNA was extracted from biopsies and cultured cells using a DNeasy Tissue Kit (Qiagen, Valencia, CA). Total RNA was isolated cultured cells using TRIzol reagent (Invitrogen, Carlsbad, CA). DNAs and RNAs were stored at $-80^{\circ} \mathrm{C}$ before analysis.
Bisulfite treatment and real-time quantitative methylation-specific PCR. DNA was treated with bisulfite to convert unmethylated cytosines to uracils prior to qMSP, as described previously ${ }^{19}$. Promoter methylation levels of $M A L$ were determined with the ABI 7900 Sequence Detection System (Applied Biosystems, Foster City, CA), using primers and probes as described previously ${ }^{20}$. A standard curve was generated using serial dilutions of CpGenome Universal Methylated DNA (CHEMICON, Temecula, CA). The NMV was defined as follows: NMV $=(M A L-S / M A L-F M)$ / (ACTB-S/ACTB-FM), where $M A L-S$ and $M A L-F M$ represent the methylation levels of $M A L$ in sample and universal methylated DNAs, respectively, while ACTB-S and $A C T B-F M$ correspond to $\beta$-Actin in sample and universal methylated DNAs, respectively ${ }^{21}$

Real-time quantitative reverse-transcriptase polymerase chain reaction. To determine $M A L$ mRNA levels, one-step real-time quantitative reverse-transcriptase polymerase chain reaction (qRT-PCR) was performed using a Qiagen QuantiTect Probe RT-PCR Kit (Qiagen, Hilden, Germany) and the ABI 7900 Sequence Detection System (Applied Biosystems, Foster City, CA). Primers and probes were the same as previously reported ${ }^{20} . \beta$-Actin was used for normalization of data. A standard curve was generated using serial dilutions of qPCR Reference Total RNA (Clontech, Mountainview, CA). The NRV was calculated according to the following formula for relative expression of target mRNA: NRV $=(\operatorname{TarS} / \operatorname{Tar} C) /(A C T B-S / A C T B-C)$, where TarS and TarC represent levels of mRNA expression for the target gene in sample and control mRNAs, respectively, whereas $A C T B-S$ and $A C T B-C$ correspond to amplified $\beta$-Actin levels in sample and control mRNAs, respectively ${ }^{21}$.

5-Aza-dC treatment of esophageal cancer cell lines. To determine whether $M A L$ inactivation was due to promoter hypermethylation in esophageal cancer, OE33 EAC cells were subjected to 5-Aza-dC (Sigma, St. Louis, MO) treatment as previously described $^{21}$. Briefly, $1 \times 10^{5}$ cells $/ \mathrm{ml}$ were seeded onto a $100 \mathrm{~mm}$ dish and grown for $24 \mathrm{~h}$. Then, 1 ul of $5 \mathrm{mM} 5$-Aza-dC per ml of cells was added every 24 hours for 6 days. DNA and RNA were harvested on day 4.

Data analysis and statistics. ROC curve analysis ${ }^{39}$ was performed using NMVs for the 67 EAC, 26 ESCC and 67 NE by Analyse-it software (Version 1.71, Analyse-it Software, Leeds, UK). Using this approach, the AUROC yielded optimal sensitivity and specificity to distinguish normal from malignant esophageal tissues, and corresponding NMV thresholds were calculated for $M A L$. The cutoff value determined from this ROC curve was applied to determine the frequency of $M A L$ methylation in each tissue type included in the present study. For all other tests, Statistica (version 6.1; StatSoft, Inc., Tulsa, OK) was used. Differences with $\mathrm{p}<0.05$ were deemed significant.

1. Bray, F., Ren, J. S., Masuyer, E. \& Ferlay, J. Global estimates of cancer prevalence for 27 sites in the adult population in 2008. Int J Cancer 132, 1133-1145 (2013).

2. Zhang, X. M. \& Guo, M. Z. The value of epigenetic markers in esophageal cancer. Front Med China 4, 378-84 (2010).

3. Enzinger, P. C. \& Mayer, R. J. Esophageal cancer. N Engl J Med 349, 2241-52 (2003).

4. Alonso, M. A. \& Weissman, S. M. cDNA cloning and sequence of MAL, a hydrophobic protein associated with human T-cell differentiation. Proc Natl Acad Sci U S A 84, 1997-2001 (1987).

5. Kim, T., Fiedler, K., Madison, D. L., Krueger, W. H. \& Pfeiffer, S. E. Cloning and characterization of MVP17: a developmentally regulated myelin protein in oligodendrocytes. J Neurosci Res 42, 413-22 (1995).

6. Schaeren-Wiemers, N., Valenzuela, D. M., Frank, M. \& Schwab, M. E. Characterization of a rat gene, rMAL, encoding a protein with four hydrophobic domains in central and peripheral myelin. J Neurosci 15, 5753-64 (1995).

7. Zacchetti, D., Peranen, J., Murata, M., Fiedler, K. \& Simons, K. VIP17/MAL, a proteolipid in apical transport vesicles. FEBS Lett 377, 465-9 (1995).

8. Martin-Belmonte, F., Kremer, L., Albar, J. P., Marazuela, M. \& Alonso, M. A. Expression of the MAL gene in the thyroid: the MAL proteolipid, a component of glycolipid-enriched membranes, is apically distributed in thyroid follicles. Endocrinology 139, 2077-84 (1998).

9. Martin-Belmonte, F., Puertollano, R., Millan, J. \& Alonso, M. A. The MAL proteolipid is necessary for the overall apical delivery of membrane proteins in the polarized epithelial Madin-Darby canine kidney and fischer rat thyroid cell lines. Mol Biol Cell 11, 2033-45 (2000).

10. Martin-Belmonte, F., Arvan, P. \& Alonso, M. A. MAL mediates apical transport of secretory proteins in polarized epithelial Madin-Darby canine kidney cells. J Biol Chem 276, 49337-42 (2001).

11. Marazuela, M. \& Alonso, M. A. Expression of MAL and MAL2, two elements of the protein machinery for raft-mediated transport, in normal and neoplastic human tissue. Histol Histopathol 19, 925-33 (2004).

12. Lind, G. E. et al. Hypermethylated MAL gene - a silent marker of early colon tumorigenesis. J Transl Med 6, 13 (2008).

13. Mimori, K. et al. MAL gene expression in esophageal cancer suppresses motility, invasion and tumorigenicity and enhances apoptosis through the Fas pathway. Oncogene 22, 3463-71 (2003).

14. Horne, H. N. et al. Inactivation of the MAL gene in breast cancer is a common event that predicts benefit from adjuvant chemotherapy. Mol Cancer Res 7, 199-209 (2009). 
15. Overmeer, R. M. et al. Repression of MAL tumour suppressor activity by promoter methylation during cervical carcinogenesis. J Pathol 219, 327-36 (2009).

16. Kuriakose, M. A. et al. Selection and validation of differentially expressed genes in head and neck cancer. Cell Mol Life Sci 61, 1372-83 (2004).

17. Wang, J. S. et al. DNA promoter hypermethylation of p16 and APC predicts neoplastic progression in Barrett's esophagus. Am J Gastroenterol 104, 2153-60 (2009).

18. Hennessy, T. P. Cancer of the oesophagus. Postgrad Med J 72, 458-63 (1996).

19. Jin, Z. et al. A multicenter, double-blinded validation study of methylation biomarkers for progression prediction in Barrett's esophagus. Cancer Res 69, 4112-5 (2009).

20. Mori, Y. et al. A genome-wide search identifies epigenetic silencing of somatostatin, tachykinin-1, and 5 other genes in colon cancer. Gastroenterology 131, 797-808 (2006)

21. Jin, Z. et al. Hypermethylation of tachykinin-1 is a potential biomarker in human esophageal cancer. Clin Cancer Res 13, 6293-300 (2007).

22. Tischoff, I. et al. Methylation of SOCS-3 and SOCS-1 in the carcinogenesis of Barrett's adenocarcinoma. Gut 56, 1047-53 (2007).

23. Hahn, H. P. et al. Intestinal differentiation in metaplastic, nongoblet columnar epithelium in the esophagus. Am J Surg Pathol 33, 1006-15 (2009).

24. Donahue, D. \& Navab, F. Significance of short-segment Barrett's esophagus. J Clin Gastroenterol 25, 480-4 (1997)

25. Lind, G. E., Ahlquist, T. \& Lothe, R. A. DNA hypermethylation of MAL: a promising diagnostic biomarker for colorectal tumors. Gastroenterology 132, 1631-2; author reply 1632 (2007).

26. Mimori, K. et al. Loss of MAL expression in precancerous lesions of the esophagus. Ann Surg Oncol 14, 1670-7 (2007).

27. Lin, S. Y. et al. Promoter CpG methylation of caveolin-1 in sporadic colorectal cancer. Anticancer Res 24, 1645-50 (2004).

28. Hvid-Jensen, F., Pedersen, L., Drewes, A. M., Sorensen, H. T. \& Funch-Jensen, P. Incidence of adenocarcinoma among patients with Barrett's esophagus. $\mathrm{N} \mathrm{Engl} \mathrm{J}$ Med 365, 1375-83 (2011).

29. Clark, G. W. et al. Short-segment Barrett's esophagus: A prevalent complication of gastroesophageal reflux disease with malignant potential. J Gastrointest Surg 1, 113-22 (1997).

30. Csendes, A. et al. Clinical, endoscopic, and functional studies in 408 patients with Barrett's esophagus, compared to 174 cases of intestinal metaplasia of the cardia. Am J Gastroenterol 97, 554-60 (2002).

31. Yagi, K. et al. Endoscopic findings of adenocarcinoma arising from short-segment Barrett's esophagus. J Gastroenterol Hepatol 19, 940-3 (2004).

32. May, A. et al. Intraepithelial high-grade neoplasia and early adenocarcinoma in short-segment Barrett's esophagus (SSBE): curative treatment using local endoscopic treatment techniques. Endoscopy 34, 604-10 (2002).

33. Hirota, W. K. et al. Specialized intestinal metaplasia, dysplasia, and cancer of the esophagus and esophagogastric junction: prevalence and clinical data. Gastroenterology 116, 277-85 (1999).
34. Hage, M. et al. Oesophageal cancer incidence and mortality in patients with longsegment Barrett's oesophagus after a mean follow-up of 12.7 years. Scand J Gastroenterol 39, 1175-9 (2004).

35. Zaninotto, G. et al. Long-term follow-up of Barrett's epithelium: medical versus antireflux surgical therapy. J Gastrointest Surg 16, 7-14; discussion 14-5 (2012).

36. Rudolph, R. E. et al. Effect of segment length on risk for neoplastic progression in patients with Barrett esophagus. Ann Intern Med 132, 612-20 (2000).

37. Lee, P. S. et al. Elevated MAL expression is accompanied by promoter hypomethylation and platinum resistance in epithelial ovarian cancer. Int J Cancer 126, 1378-89 (2010).

38. Cao, W. et al. Epigenetic silencing of MAL, a putative tumor suppressor gene, can contribute to human epithelium cell carcinoma. Mol Cancer 9, 296 (2010).

39. Hanley, J. A. \& McNeil, B. J. The meaning and use of the area under a receiver operating characteristic (ROC) curve. Radiology 143, 29-36 (1982).

\section{Acknowledgements}

Supported by National Nature Science Foundation of China grant 81172282,Natura Science Foundation of SZU grants 201108 and T201202 to Z Jin; NIH grants DK087454, CA146799 and CA133012 to SJ Meltzer; National Nature Science Foundation of China grant 81171921 and the Science and Technology Bureau of ShenZhen City grant JC201006010727A and JCYJ20120613165853326 to L Wang.

\section{Author contributions}

Dr. Liang Wang as L.W., Dr. Yuan Zhang as Y. Z., Dr. Yulan Cheng as Y.C., Dr. Yan Gao as Y.G., Dr. Xianling Feng as X.Fe., Dr. Ming Dong as M.D., Dr. Ziyi Cao as Z.C., Dr. Si Chen as S.C., Dr. Huimin Yu as H.Y., Dr. Zhenfu Zhao as Z.Z., Dr. Xiaojing Zhang as X.Z., Dr. Jie Liu as J.L., Dr. Xinmin Fan as X.Fa., Dr. Yuriko Mori as Y.M., Dr. Stephen J. Meltzer as S.J.M., Prof. Zhe Jin as Z.J., Z.J. and S.J.M. designed the study. Z.J. and L.W. wrote the main manuscript text. Y.Z., Y.C., Y.G., X.Fe., M.D., Z.C., S.C., H.Y., Z.Z., X.Z., J.L., X.Fa. and Y.M analyzed and interpreted the data. All authors reviewed the manuscript.

\section{Additional information}

Competing financial interests: The authors declare no competing financial interests.

How to cite this article: Jin, Z. et al. MAL hypermethylation is a tissue-specific event that correlates with MAL mRNA expression in esophageal carcinoma. Sci. Rep. 3, 2838; DOI:10.1038/srep02838 (2013)

This work is licensed under a Creative Commons AttributionNonCommercial-NoDerivs 3.0 Unported license. To view a copy of this license, visit http://creativecommons.org/licenses/by-nc-nd/3.0 